\section{Proposal to put Prestel viewdata system into every British home}

\section{London}

BRITISH Telecom has decided to embark on an ambitious scheme to put its pioneering viewdata system Prestel into every telephone subscriber's household. At present. only 24,000 British households have Prestel terminals, out of 18 million domestic telephone subscribers. It is understood that British Telecom will in the first instance set up a trial, with subscribers in one London telephone district and one district outside London being supplied with Prestel terminals for a fixed time. The town of Milton Keynes, a traditional test-bed for British Telecom's innovations and with the country's youngest, least technology-shy population, is being tipped as a possible trial district, although a city such as Nottingham would be more representative.

British Telecom has not yet publicly announced its plans, and the size of the trial sample is not known. It is understood that tenders have been invited for the supply of 5 million Prestel terminals, and Philips Business Systems is one of the companies invited to tender. Officials want the trial to start by September.

Launched in 1979, Prestel has never lived up to its early promise, and British Telecom's hope that the Prestel terminal would become as ubiquitous as the television set or refrigerator failed to become reality. Prestel was originally conceived as a means of increasing domestic telephone traffic, traditionally subsidized by the business community, but soon became established as a trade medium when the ferry company Sealink installed 600 terminals free of charge in travel agencies

to supply continuously updated information on ferry availability. Indeed, by 1981 , British Telecom had changed its marketing strategy to aim at the business world rather than householders. Now, of Prestel's 78,000 terminals, 69 per cent are in the workplace and 31 per cent in the home.

The lack of interest of domestic users is generally blamed on poor marketing and the cost of hardware. At present, the least expensive means of using Prestel is to buy an adaptor priced at around $£ 100$ for an existing television set. Although Prestel's office says the system makes a profit (figures are not made public), of the numerous services available, it is the financial and travel companies that supply the bulk of the business.

Despite the relatively modest number of Prestel subscribers, the service is growing. The number of terminals has increased more than threefold since 198283 and in 1981 there were only 1,300 domestic users. If British Telecom does decide to provide householders with free Prestel sets, the capital outlay will be considerable. But one immediate benefit would be to relieve the much-lambasted directory service (the Prestel system would provide a national telephone directory), which is now free, although British Telecom hints that this might not always be so.

The overall standard of service from British Telecom has come in for a good deal of criticism recently. Its Prestel scheme could do much for public relations, and with a half-year pre-tax profit to September this year of $£ 1,120$ million, it should be able to take the financial strain.

Simon Hadlington

\section{Seismologists unshaken by earthquake}

\section{Washington}

Two more earthquakes hit southern California last week, both measuring a little more than six on the Richter scale. But unlike the earthquake of similar magnitude that rattled Los Angeles on 1 October, no deaths were caused and few puzzles were set for seismologists.

Robert Wesson, a geophysicist specializing in earthquake prediction at the United States Geological Survey (USGS), said that while the 1 October earthquake "made us feel humble, this one made us feel that we are beginning to understand what is going on". The pair of earthquakes occurred on the Superstition Hills fault in the sparsely populated Imperial Valley close to the Mexican border.

The area's location at the end of the San Andreas fault system puts it at risk from earthquakes. Over the past decade, there has been a pattern in the place and time of Californian earthquakes that, while not fully understood, suggests that stress, generated in the slow movement of the Pacific plate, is transferred up and down the faults in a predictable manner. Stress is particularly likely to concentrate at the end of faults which are slowly creeping. Superstition Hills had been marked down by USGS researchers as a possible spot for an earthquake during 1986-96. Earthquakes occurred nearby in 1979 and 1980.

The citizens of Los Angeles are not likely to gain much comfort from the knowledge that long-term earthquake prediction is gaining some respectability. Similar studies by the USGS suggest that there is a $\mathbf{5 0}$ per cent chance of a magnitude 8 earthquake, likely to cause 3,000-13,000 deaths, on the San Andreas fault in the next 30 years.

\section{Racing for the cosmic flash}

\section{Sydney}

Astronomers in Australia and New Zealand are rushing to complete gammaray telescopes in time to catch the expected arrival of gamma rays from the February supernova in the Large Magellanic Cloud.

Two new instruments are under construction; one in the Australian desert at Woomera and the other $1,650 \mathrm{~m}$ up a mountain near Blenheim on New Zealand's South Island.

Visually, the supernova has long passed its peak, but no gamma rays have yet been seen because they cannot penetrate the shell of hot gas and dust that surrounds the supernova's innermost regions. But that veil is growing thinner.

Gamma-ray telescopes observe indirectly. When gamma rays strike the atoms in the upper atmosphere, they scatter their energy in a cascade of secondary particles; these in turn give off flashes of light called Cerenkov radiation which can be picked up by the telescope.

The Woomera instrument, being built by the University of Adelaide's department of physics at a cost of $A \$ 220,000$, should be able to locate a light source to within a half degree on the sky. The telescope consists of 57 separate $80-\mathrm{cm}$ diameter mirrors mounted on a steerable frame in three groups of 19 . In each group, the light collected by the mirrors is brought to a common focus where it is picked up by a photo-electric detector.

The detectors can resolve time differences as small as five thousand millionths of a second. Any event not detected simultaneously at the three focal points is rejected as noise.

The Woomera site was chosen for its dark skies, the prime criterion for gammaray observation. The telescope had been planned long before the supernova and was not due for completion until the middle of next year. But the project leader, Professor John Prescott, now plans to have it operating by 1 January.

The New Zealand telescope owes its genesis entirely to the supernova. Universities in Japan, Australia and New Zealand are working together, with Professor Y. Murakami of the University of Tokyo as the project leader. According to Dr John Humble of the University of Tasmania, the telescope is largely being put together using spare parts gathered from around Japan. New Zealand is providing the site and Australia largely 'moral support'. The site was chosen in May, construction began in September and the first test observation had been completed by 20 November.

Charles Morgan 